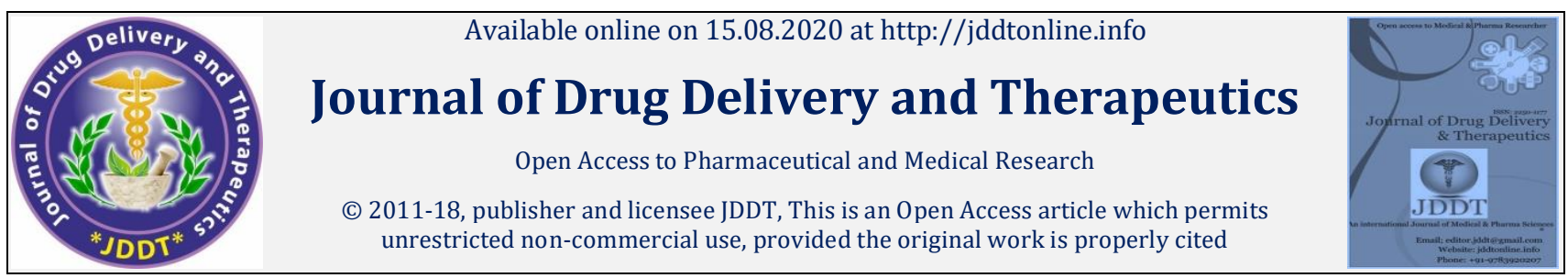

Open $\odot$ Access

Mini Review

\title{
On Buprenorphine Implants: Should It Be Widely Used For Medically Assisted Treatment?
}

\section{Patrick Bailey}

\begin{abstract}
Buprenorphine implants are recognized as one of the routes to administer Medically Assisted Treatment (MAT). However, the use of these implants is far less compared to oral medications in battling substance abuse. This article explores the likely reasons why buprenorphine implants are not as commonly recommended, and whether it is a feasible option to consider it a mainstream type of treatment.
\end{abstract}

Article Info: Received 08 July 2020; Review Completed 25 July 2020; Accepted 08 August 2020; Available online 15 August 2020

Cite this article as:

Bailey P, On Buprenorphine Implants: Should It Be Widely Used For Medically Assisted Treatment?, Journal of Drug Delivery and Therapeutics. 2020; 10(4-s):214-216 http://dx.doi.org/10.22270/jddt.v10i4-s.4257

*For Correspondence:

Patrick Bailey. Email: p.bailey@patrickbaileys.com

\section{Introduction}

Medically Assisted Treatment (MAT) has gained a solid footing when it comes to effectiveness in substance abuse rehabilitation. The use of prescription drugs such as naloxone, buprenorphine, or methadone for drug or alcohol addiction yielded positive results with many patients being able to abstain from their substance of choice. However, most MAT approaches involve oral intake of these medications. Buprenorphine implants, which were recently approved by the FDA in 2016, are relatively new to the market as compared to its oral drug counterparts.

Despite this approval, many treatment centers retain their medical detoxification services through the use of prescription drugs taken orally. As a result, patients who go through addiction rehabilitation may not be aware that they can use buprenorphine implants to treat opioid use disorder. It is important to understand that this is just one angle of the story. Buprenorphine implants have not received mainstream attention, and this article aims to explore the reasons why. Secondly, this article also aims to uncover if buprenorphine implants are a viable option for those seeking lasting recovery from substance abuse.

\section{Understanding Buprenorphine Implants}

To further understand buprenorphine implants, it is essential to know the nature of the drug buprenorphine. Buprenorphine is recognized as an opioid medication primarily used to treat opioid addiction. This drug differs from other sub-types of opioids as it is considered a partial opioid agonist. Partial opioid agonists are recognized for presenting these following effects:
1. Fewer feelings of euphoria

2. Decreased cravings

3. Lesser physical dependency

4. Lower risk of misuse

5. Blocked effects of other opioids

The main goal of using buprenorphine is to dampen the withdrawal symptoms of opioid abuse while helping patients stay in treatment. The oral route of the drug was approved in 2002 under the brand names Subutex and Suboxone.

Over the years, modern medicine has made it possible for many prescription drugs to be administered using a delayedrelease or sustained-release system. The use of implants is highly advertised in the field of birth control such as Implanon and Nexplanon. The effectiveness ratings of these implanted drugs, which are claimed to be around 99\%, had several researchers and pharmaceutical companies apply the same technology with addiction treatment. Hence, the buprenorphine implant medication was conceived. The benefits of taking buprenorphine via implanted route are:

1. Consistent release of the correct dosage, preventing overdose

2. Avoiding the risk of misuse

3. Scheduled release, preventing relapses in addiction recovery

The high level of structure and control that buprenorphine implants offer are beneficial to people who desire effective 
MAT approaches. Whether an individual is considering rehab for alcohol or drug abuse, having referrals for buprenorphine or MAT drug implants can be another way to explore other potentially successful options.

\section{Why Buprenorphine Implants are Not Widely Used}

Even is a drug is scientifically backed with research on its effectiveness, there are still challenges that hinder it from receiving mainstream acceptance. Such is the case of buprenorphine implants. There are many reasons why this is so; which stems from three perspectives: The angle of patients, healthcare providers, and the nature of the drug itself.

a.) From a patient perspective: From a patient's perspective, having an implant means you have to undergo an invasive procedure. This requires placing the drug subcutaneously or performing minor surgery to place implants close to where drug absorption can take place. A study published in NCBI revealed that $80 \%$ of patients prefer a non-invasive or minimally invasive approach to treatment. There is a perception that exists--more invasive procedures equals more risk. If oral medication is available, patients are more inclined to try that particular route initially.

b.) From a healthcare provider perspective: Most healthcare providers for addiction treatment are doctors and specialists within a drug or alcohol treatment center. Not all doctors and addiction specialists are trained to place buprenorphine implants, which makes them less likely recommended as the first line of care. Most buprenorphine implant recommendations are set as referrals, which could take up time and not appealing to those who are seeking immediate care.

Another potential reason why many healthcare experts do not readily recommend buprenorphine implants is to decrease liability. Since there are life-threatening risks to these implants, doctors and addiction specialists may be reluctant in listing this drug as the main mode of MAT.

c.) From the nature of the drug: Probuphine, the brand name commonly associated with buprenorphine implants, have medical disclaimers explaining the risk of using the drug. According to the website, there are serious complications that could happen such as nerve or blood vessel injury, implant migration causing organ blockage, or expulsion of the drug. Any of these serious complications, according to the disclaimer, can potentially cause death. For patients to consider this treatment and for healthcare providers to recommend buprenorphine implants as the first line of care, a thorough assessment of the benefits should greatly outweigh these risks.

Aside from the perspective mentioned above, another probable reason for this less-popular mode of treatment is its price point. One implant costs approximately $\$ 1,294$, which is a hefty amount compared to 30 units of Suboxone which is around \$572. To add to this, the 2016 FDA approval of the drug gives the impression that it is less mainstream, having been around for public use for 4 years as of writing.

\section{Should Buprenorphine Implants be the First Line of Care?}

Another question to be explored is if buprenorphine implants should be considered as the first line of treatment under MAT. Considering the risks, price point, and patient perspectives mentioned above, it is possible to present buprenorphine implants as a viable option for treatment and should be discussed equally as other MAT options.
There are individuals who have difficulties maintaining a drug intake schedule especially if it is a self-directed task. Another reason for recommending buprenorphine implants is to avoid misuse. Cases of buprenorphine overdose have happened especially when used in tandem with other opioids or depressant drugs. To provide the best type of treatment, a solution would be to have a comprehensive evaluation of each patient. Each evaluation should have laboratory testing that determines the severity of substance abuse, as well as a questionnaire that explores the treatment preferences of each patient. Programs for alcohol or drug addiction should be customized enough such that specialists are able to target the root cause of substance abuse for each individual.

\section{Conclusion}

In summary, buprenorphine implants are not commonly recommended because of several reasons. Various perspectives are considered, such as those coming from the patient, treatment facilities, experts, and the nature of the drug. Reasons such as being an invasive procedure, price points, and potential risks may make decision-makers choose the other options available perceived as mainstream.

Medically Assisted Treatment, like other substance abuse approaches, should have a variety of sub-procedures available for the patient to choose from. Whether it's through oral, intravenous, or implanted medication, a custom-fit principle must be applied if the individual chooses MAT as their primary mode of treatment. Buprenorphine implants as an option should be explained thoroughly. If chosen, patients must have the right to know the benefits, risks, and instructions for use.

\section{References}

1. McKeganey N, Russell C, Cockayne L. Medically assisted recovery from opiate dependence within the context of the UK drug strategy: Methadone and Suboxone (buprenorphinenaloxone) patients compared. Journal of substance abuse treatment. 2013 Jan 1;44(1):97-102. Retrieved from https://www.sciencedirect.com/science/article/pii/S074054 7212000803

2. US Food and Drug Administration. FDA approves the first buprenorphine implant for the treatment of opioid dependence. News Release. Silver Spring, MD: FDA. 2016 May 26. Retrieved from https://www.fda.gov/news-events/pressannouncements/fda-approves-first-buprenorphine-implanttreatment-opioid-dependence

3. Food and Drug Administration. Subutex and Suboxone approved to treat opiate dependence. FDA Talk Paper T02-38. Retrieved February. 2002 Oct 8;19:2003. Retrieved from https://www.naabt.org/faq_answers.cfm?ID=2

4. Stewart SA, Domínguez-Robles J, Donnelly RF, Larrañeta E. Implantable polymeric drug delivery devices: classification, manufacture, materials, and clinical applications. Polymers. 2018 Dec;10(12):1379. Retrieved from https://www.mdpi.com/2073-4360/10/12/1379

5. White J, Bell J, Saunders JB, Williamson P, Makowska M, Farquharson A, Beebe KL. Open-label dose-finding trial of buprenorphine implants (Probuphine) ${ }^{\circledR}$ for treatment of heroin dependence. Drug and alcohol dependence. 2009 Jul 1;103(1-2):37-43. Retrieved from Open-label dose-finding trial of buprenorphine implants (Probuphine)® for treatment of heroin dependence

6. Bell JR, Butler B, Lawrance A, Batey R, Salmelainen P. Comparing overdose mortality associated with methadone and buprenorphine treatment. Drug and alcohol dependence. 2009 Sep 1;104(1-2):73-7. Retrieved from https://www.sciencedirect.com/science/article/abs/pii/S03 $7687160900115 \mathrm{X}$ 


\section{About the Author:}

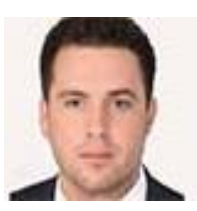

\section{PATRICK BAILEY}

Patrick Bailey is a professional writer mainly in the fields of mental health, addiction, and living in recovery. He attempts to stay on top of the latest news in the addiction

Author's Webpage: http://patrickbaileys.com/my-guest-posts/ and the mental health world and enjoy writing about these topics to break the stigma associated with them. 